\title{
Exceeding the Asymptotic Limit of Polymer Drag Reduction
}

\author{
George H. Choueiri, Jose M. Lopez, and Björn Hof ${ }^{\dagger}$ \\ IST Austria, 3400 Klosterneuburg, Austria
}

(Received 9 June 2017; revised manuscript received 22 December 2017; published 19 March 2018)

\begin{abstract}
The drag of turbulent flows can be drastically decreased by adding small amounts of high molecular weight polymers. While drag reduction initially increases with polymer concentration, it eventually saturates to what is known as the maximum drag reduction (MDR) asymptote; this asymptote is generally attributed to the dynamics being reduced to a marginal yet persistent state of subdued turbulent motion. Contrary to this accepted view, we show that, for an appropriate choice of parameters, polymers can reduce the drag beyond the suggested asymptotic limit, eliminating turbulence and giving way to laminar flow. At higher polymer concentrations, however, the laminar state becomes unstable, resulting in a fluctuating flow with the characteristic drag of the MDR asymptote. Our findings indicate that the asymptotic state is hence dynamically disconnected from ordinary turbulence.
\end{abstract}

DOI: 10.1103/PhysRevLett.120.124501

In pipe and channel flows, turbulence is often responsible for more than $90 \%$ of the friction losses. A very efficient and often used method to reduce this frictional drag is by addition of small amounts of long chain polymers. Since its discovery nearly 70 years ago, this effect has been studied extensively and various theories have been put forward to explain the mechanism of drag reduction (DR). It is commonly found that DR increases with polymer concentration but eventually saturates to the "maximum drag reduction" (MDR) asymptote [1], as shown in Fig. 1(a). All studies to date suggest that, regardless of polymer concentration, this limit cannot be overcome [2-4]. The MDR asymptote is found to be identical for different types of polymers and polymer solvent combinations [1]. Based on these observations, the general view is that polymers decrease turbulent activity (either via elastic [5] or viscous effects [6] or both) and that, eventually, turbulence is reduced to a marginal state which corresponds to the MDR asymptote. More explicitly, it has been argued $[4,7,8]$ that turbulence is minimized to the edge between laminar and turbulent motion. It is, however, not clear how turbulence can persist in this limit. In purely Newtonian flow, the edge is intrinsically unstable and separates initial conditions that go turbulent from those that collapse back to laminar, and it has not been shown if or how this state would become stable due to the action of polymers.

An alternative interpretation of the MDR state was given more recently by Samanta et al. [9], where it has been observed that, with increasing polymer concentration, a different instability sets in; due to its occurrence at finite inertia and the elastic nature of the polymers, it has been dubbed elastoinertial instability. This instability has been observed independently in direct numerical simulations [10] and in laboratory experiments [9]. In the experimental study [9], it could be shown that the elastoinertial instability strictly arises at Reynolds numbers below those at which the MDR asymptote is assumed and that, at the same time, the transition to turbulence is delayed. Also, it has been observed that, for moderately high polymer concentrations, chaotic motion sets in at Reynolds numbers $\left(\operatorname{Re}=U_{b} D / \nu\right)$ $\approx 900$, much below those at which turbulence can be observed in Newtonian fluids $(\operatorname{Re} \approx 2000)$; this is in qualitative agreement with earlier studies [11,12]. Here, $U_{b}$ is the bulk flow velocity, $D$ is the pipe diameter, and $\nu$ is the kinematic viscosity of the fluid. Based on these observations, Samanta et al. [9] proposed that on MDR the dynamics are driven by the elastoinertial instability, while Newtonian turbulence (NT) is eliminated before reaching MDR. The authors dubbed the corresponding dynamical state elastoinertial turbulence (EIT).

In the following we will demonstrate that polymers can, for an appropriate choice of parameters, eliminate fully turbulent motion. For increasing polymer concentrations, the laminar flow eventually becomes unstable again, giving rise to the MDR state, which is hence disconnected from NT. As will be shown, flows in the asymptotic drag limit at high Reynolds numbers structurally differ from NT and, at the same time, closely agree with the characteristic streak patterns resulting from EIT at Reynolds numbers well below the threshold for NT.

Experiments are carried out for pipe flow with water as the solvent. A concentrated polymer solution was injected into the water at the pipe entrance. Dye was added to the concentrated polymer solution to verify that the fluid was uniformly mixed and homogeneous well upstream of the first measurement station located $480 \mathrm{D}$ downstream (see the Supplemental Material [13] for more details on the experimental setup). In the first set of experiments, the Reynolds number was held fixed at 5200, starting from Newtonian flow, where the friction factor is found to agree with the 

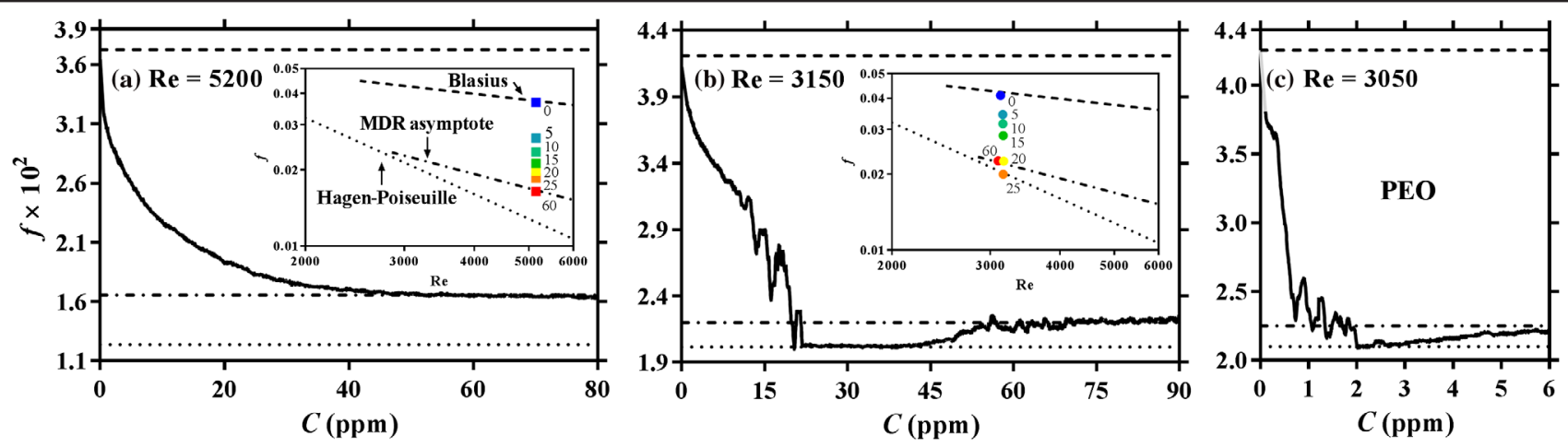

FIG. 1. In (a) and (b), the main figures show the Darcy-Weisbach friction factor $f$, as a function of continuously increasing polyacrylamide (PAM) concentration, while the insets show $f$ at distinct concentrations for a constant Reynolds number. Dashed lines, Blasius friction factor scaling $\left(f=0.316 \mathrm{Re}^{-1 / 4}\right)$; dotted lines, Hagen-Poiseuille flow ( $\left.f=64 / \mathrm{Re}\right)$; dashed-dotted lines, MDR asymptote $\left(f=2.36 \mathrm{Re}^{-0.58}\right)$. (c) The use of polyethylene oxide (PEO) results in the same qualitative behavior as observed in (b).

Blasius correlation for friction factors. When the concentration, $C$, is increased in distinct steps from 0 parts per million (ppm), by weight, to a maximum of $60 \mathrm{ppm}$, the friction factor is observed to monotonically decrease and eventually settle on the maximum drag reduction asymptote [inset of Fig. 1(a)]. Note that the addition of polymers causes a viscosity increase in the fluid which has been measured and is taken into account for the quoted Reynolds number values for all of the measurements reported. When the experiment is repeated and $C$ is increased linearly from 0 to $80 \mathrm{ppm}$ [over a time span of $\approx 60000$ advective time units in a quasisteady fashion; Fig. 1(a)], the continuous decrease of the friction factor and the monotonic approach towards MDR [48 ppm; Fig. 1(a)] are clearly seen. This observation precisely complies with the standard picture of polymer drag reduction and its asymptotic limit (see Fig. 3 in Ref. [3]).

A very different scenario is observed when Re is set to 3150 [inset of Fig. 1(b)]. Again beginning on the Blasius friction factor line for NT, an increase in polymer concentration to $20 \mathrm{ppm}$ appears to reduce the friction factor, on average, to the predicted MDR value. However, a further increase to $25 \mathrm{ppm}$ pushes the friction factor below what was believed to be the limiting threshold in polymer drag reduction and recovers the laminar (Hagen-Poiseuille) value. In addition, measured fluctuation levels drop to the level of instrument noise recorded at zero flow. A further increase in polymer concentration surprisingly destabilizes the laminar flow; fluctuations increase and the friction factor increases to the "maximum" drag reduction asymptote [60 ppm; Fig. 1(b) inset]. While the average friction factors for 20 and $60 \mathrm{ppm}$ are almost identical and comply with the MDR value, structurally, the flows are very different [compare Fig. 2(b) to Figs. 2(e) and 2(f), where the red and blue marked regions strongly deviate from the mean streamwise speed, i.e., streaks]. At $20 \mathrm{ppm}$ the flow is intermittent, consisting of localized bursts of activity separated by much more quiescent regions. For $C>50 \mathrm{ppm}$, however, the entire flow is in a fluctuating state [Figs. 2(e) and 2(f)], but, notably, fluctuation levels are significantly reduced when compared to the bursts at $20 \mathrm{ppm}$. This picture is confirmed when the concentration is increased continuously at a slow rate [from 0 to $90 \mathrm{ppm}$ over the course of $\approx 60000$ advective time units; Fig. 1(b)], while Re is held fixed at $\operatorname{Re}=3150$. The friction factor decreases gradually and, close to $20 \mathrm{ppm}$, drops more steeply to the laminar flow value. Laminar flow persists up to about $40 \mathrm{ppm}$, and the friction factor then begins to increase until it settles to the MDR value for $C>55 \mathrm{ppm}$.

These experiments were repeated following two alternative protocols to ensure that the results were robust and independent of the detailed experimental procedures. In the

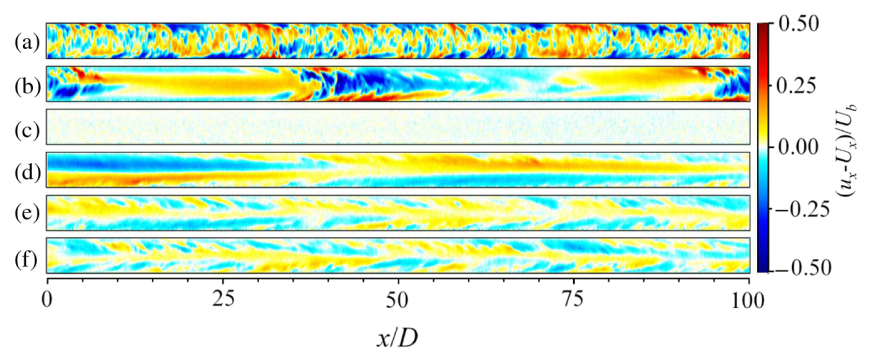

FIG. 2. Streamwise velocity deviations with respect to the mean flow profile. High-speed streaks appear in red, low-speed streaks in blue. The velocity field was obtained from particle image velocimetry (PIV) images taken in a cross-section plane $\sim 3 D$ in length. The larger structures are then reconstructed by exploiting the circumstance that turbulence is advected downstream swiftly and structural changes in time occur at a slower rate; therefore, time information can be converted to space (i.e., Taylor's frozen turbulence hypothesis). Images taken at different times are assembled and matched to regain the spatial structure. Measurements are carried out at $\mathrm{Re}=3150$ and polymer concentrations of (a) 0, (b) 20, (c) 30, (d) 50, (e) 100, and (f) $150 \mathrm{ppm}$. The figure is scaled to $20 \%$ in the horizontal direction. $u_{x}$ is the local instantaneous streamwise velocity, $U_{x}$ the average streamwise velocity, and $U_{b}$ the bulk velocity. The flow direction is from left to right. 
first case, polymers were injected $\sim 150 D$ further downstream into the fully turbulent flow, and the results obtained were identical to the above within experimental uncertainties. In the second case, as opposed to polymer injections, experiments were carried out with premixed polymer solutions of set concentrations. Equally, in this case a laminar window was found for $\mathrm{Re} \lesssim 3600$ that separates drag reduced turbulence at lower concentrations and MDR at higher ones.

Since the MDR friction scaling is a universal property of drag reducing polymers, we tested whether relaminarization prior to the approach to MDR is also found for other polymers. Experiments were carried out with polyethylene oxide (PEO) and, indeed, the same qualitative behavior is observed [Fig. 1(c)]: with low Reynolds numbers $(\operatorname{Re}=3050)$, flows fully relaminarize prior to the approach to MDR. To elucidate the qualitative difference in the drag reduction scenario between the standard case [Fig. 1(a)] and the relaminarization [Figs. 1(b) and 1(c)], we carry out a detailed investigation of the Reynolds number-polymer concentration parameter space for polyacrylamide. Specifically, we observe that the onset of turbulence (the left lower branch in Fig. 3) is delayed by the action of polymers. While in a previous study [9] the delay extended only to parameters where, in the Newtonian case, flows at most are spatiotemporally intermittent $(\operatorname{Re} \approx 2600)$, in the present case the delay extends to $\sim 25 \%$ larger Reynolds numbers where, in the Newtonian case, turbulence is space filling $(\operatorname{Re} \gtrsim 2800)$ and assumes its characteristic friction factor scaling (Blasius). For increasing Re values in this regime ( $C \lesssim 20 \mathrm{ppm}$ in Fig. 3), turbulence sets in in the form of localized turbulent structures (puffs) and, subsequently, to growing turbulent structures, commonly referred to as slugs. Like the onset of puffs, the onset of slugs is equally delayed compared to Newtonian fluids. By

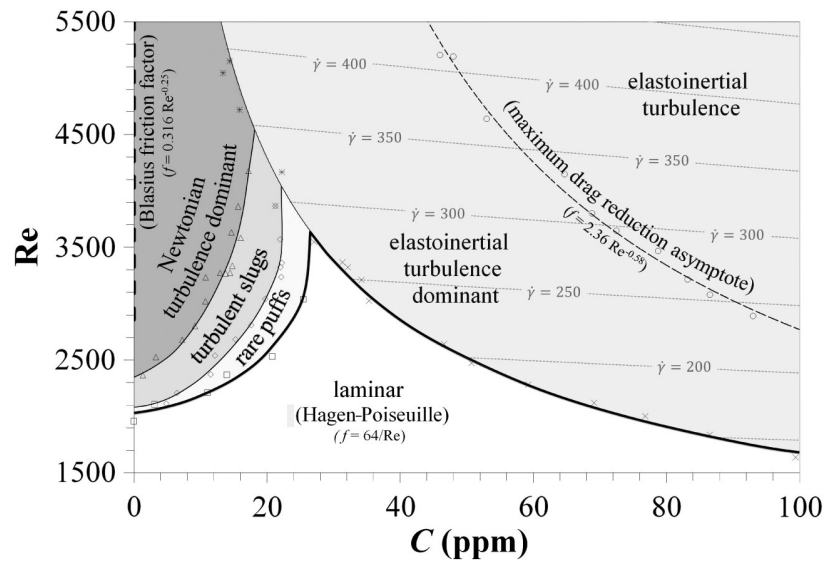

FIG. 3. State map for Reynolds number versus polymer concentration. While NT is governed by the Re, the elastoinertial instability is governed by the relation of $\dot{\gamma}$ to $C$ rather than Re to $C$, where $\dot{\gamma}=8 U / D$ is the shear rate. Lines with constant $\dot{\gamma}$ values have been superimposed in the region of EIT. contrast, at higher concentrations ( $\gtrsim 30 \mathrm{ppm})$, a qualitatively different instability is encountered. Here, fluctuations set in more uniformly in space [Fig. 2(d)], lacking the spatial intermittent character of the Newtonian transition scenario. This observation is in qualitative agreement with the previous study on elastoinertial instability [9]. For a further increase in concentration $(C \gtrsim 90 \mathrm{ppm})$, this instability occurs at Re distinctly below the lowest Re value where NT would be observed.

Starting from Newtonian turbulence $(C=0)$ for a fixed Re (Fig. 3, the Blasius friction factor line on the left) and increasing polymer concentration, if $\operatorname{Re} \leq 4500$, fully turbulent motion becomes unstable and returns to a regime of localized turbulent patches interspersed with more quiescent regions. Upon a further increase in concentration (for $\operatorname{Re} \lesssim 3600$ ), the localized turbulent structures are found to collapse and the flow completely relaminarizes. This scenario is the inverse of the familiar turbulence transition scenario in Newtonian pipe flow, where turbulence first appears in the form of localized patches (first puffs, then slugs). Fully turbulent flow becomes stable only upon a further increase in Re [14]. For even larger polymer concentrations, the laminar flow becomes unstable as "elastoinertial" instability sets in (the right lower branch in Fig. 3). In practice, this relaminarization window can only be observed for relatively low Reynolds numbers where the shear rates are sufficiently large to suppress NT but too low to trigger the polymer driven elastoinertial instability. At somewhat larger Reynolds numbers $(3600<\operatorname{Re}<4500)$, turbulence breaks up into localized puffs or slugs; however, before complete relaminarization is observed, the instability towards EIT occurs, resulting in a mixed state which then eventually approaches MDR. At higher $\operatorname{Re}$ values $(\operatorname{Re}>4500)$, the scenario seemingly follows the traditional view of MDR where NT is continuously suppressed by the action of polymers and the friction factor eventually settles to MDR. However, what has been overlooked in previous studies is that, at intermediate concentrations, elastoinertial instability sets in before MDR is reached.

The reverse transition from fully turbulent flow to localized slugs and puffs (for $\operatorname{Re}<4500$ ) and, eventually, laminar flow (for $\operatorname{Re}<3400$ ), and the fact that onset of the elastoinertial instability is always encountered prior to the final approach to MDR raises the question of whether, at larger Re, NT is also eventually fully suppressed as the polymer concentration is increased. In order to answer this question, we will in the following compare the flow structure in the MDR limit for different Reynolds numbers. When comparing flow structures between Newtonian turbulence [Fig. 2(a)] and MDR [Fig. 2(f)] for flow at $\mathrm{Re}=3150$, it is apparent that the streak pattern characteristic for NT is virtually absent in the MDR flow. In the latter case, only weak, much more elongated streaks that are slightly inclined with respect to the flow direction are 


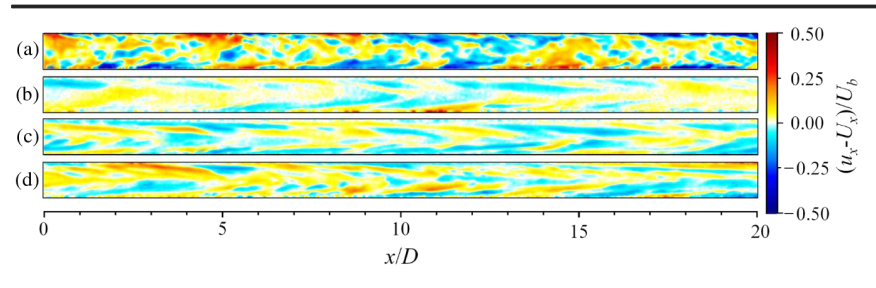

FIG. 4. Streamwise velocity deviations with respect to the mean flow profile for various Reynolds numbers and polymer concentrations. (a) $\mathrm{Re}=5200$ (0 ppm). (b) $\mathrm{Re}=1000$ (70 ppm, 50\% glycerol). (c) $\operatorname{Re}=5200$ (125 ppm). (d) $\operatorname{Re}=10000$ (68 ppm).

present. As noted above, these structures arise after NT has been eliminated and are a consequence of the elastoinertial instability, directly arising from a state of laminar flow. The MDR flow at $\mathrm{Re}=5200$ and 10000 [Fig. 4(c) and 4(d)] qualitatively resembles that at $\mathrm{Re}=3150$ [Figs. 2(e) and 2(f)]. Also, here the characteristic streak patterns of NT have disappeared, and only weak, elongated streaks are visible. Overall, the resemblance of flow structures on MDR for the three Reynolds numbers show that EIT has characteristic features that are independent of the Re. To further illustrate this point, we increased the solution viscosity using a $50 \%$ glycerol solution to trigger the elastoinertial instability at a Re below that which can sustain NT [i.e., 1000 in this case; Fig. 4(b)], and we again observe low amplitude, elongated streaks similar to those on MDR at higher Re values. Since the flows at $\operatorname{Re}=1000$ and 3150 are clearly disconnected and distinct from NT and result solely from the elastoinertial instability, we propose that, at $\operatorname{Re}=5200$ and 10000 (where MDR is approached in the usual manner), NT is also eventually marginalized - if not fully suppressed-and replaced by EIT, only that here, the eventual state is preceded by a coexistence phase rather than by relaminarization. Finally, we computed the Reynolds stresses $\overline{u^{\prime} v^{\prime}}$, where $u^{\prime}$ and $v^{\prime}$

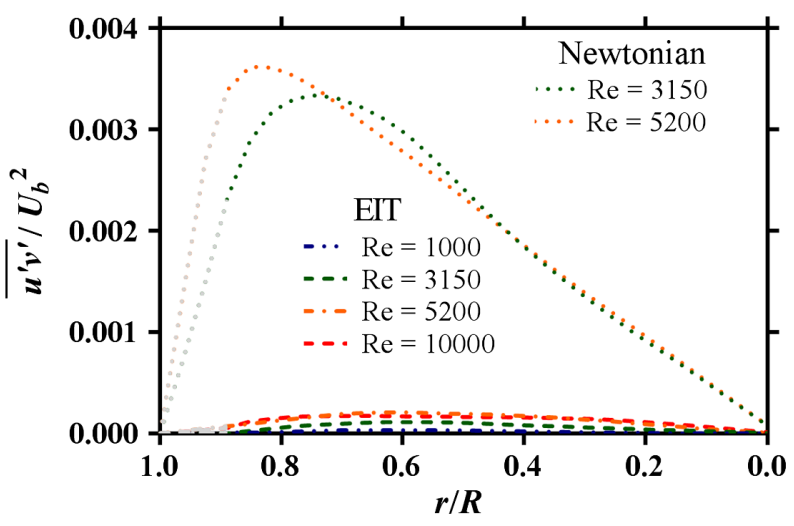

FIG. 5. Reynolds stresses for NT and EIT normalized by the square of the bulk flow velocity as a function of the radial position $r$, normalized by the pipe radius $R$. Because of the higher uncertainty in the near-wall PIV measurements, results in this region were forced to zero at the wall and are marked in gray where this was done. are the velocity fluctuations in the streamwise and radial directions, respectively. As shown in Fig. 5, flows in the EIT limit have markedly lower Reynolds stresses than their Newtonian counterparts (in agreement with earlier Reynolds stress measurements at MDR [15]). We argue that the much lower Reynolds stress level further distinguishes flows in the MDR limit as a separate dynamical state.

In summary, we show in this Letter that the drag reduction limit and the characteristic approach towards it result from the interplay between two states of turbulence, Newtonian turbulence and elastoinertial turbulence. In regions of parameter space where EIT is absent, polymers can fully relaminarize the flow and hence reduce the drag beyond the maximum limit. Conversely, in the absence of NT, an increase in polymer concentration (starting from laminar flow) leads to a drag increase and the MDR asymptote is approached from below. A familiar picture is uncovered if EIT and NT coexist. As NT becomes further suppressed, the drag reduces towards the MDR limit. With this approach, the flow structures progressively resemble those of low-Re EIT, suggesting that Newtonian turbulence is marginalized and replaced by elastoinertial turbulence, and also that the characteristic MDR friction factor scaling is a property of the latter.

The authors thank Philipp Maier and the IST Austria workshop for their dedicated technical support. The research leading to these results has received funding from the People Programme (Marie Curie Actions) of the European Union's Seventh Framework Programme (FP7/ 2007-2013) under REA Grant Agreement No. 291734 and the European Research Council under the European Union's Seventh Framework Programme (FP/20072013)/ERC Grant Agreement No. 306589.

*George.Choueiri@ist.ac.at

†bhof@ist.ac.at

[1] P. Virk, H. Mickley, and K. Smith, J. Appl. Mech. 37, 488 (1970).

[2] K. R. Sreenivasan and C. M. White, J. Fluid Mech. 409, 149 (2000).

[3] C. M. White and M. G. Mungal, Annu. Rev. Fluid Mech. 40, 235 (2008).

[4] L. Xi and M. D. Graham, Phys. Rev. Lett. 108, 028301 (2012).

[5] M. Tabor and P. De Gennes, Europhys. Lett. 2, 519 (1986).

[6] J. L. Lumley, Annu. Rev. Fluid Mech. 1, 367 (1969).

[7] I. Procaccia, V. S. Lvov, and R. Benzi, Rev. Mod. Phys. 80, 225 (2008).

[8] L. Xi and M. D. Graham, Phys. Rev. Lett. 104, 218301 (2010).

[9] D. Samanta, Y. Dubief, M. Holzner, C. Schäfer, A. N. Morozov, C. Wagner, and B. Hof, Proc. Natl. Acad. Sci. U.S.A. 110, 10557 (2013).

[10] Y. Dubief, V. E. Terrapon, and J. Soria, Phys. Fluids 25, 110817 (2013). 
[11] A. Ram and A. Tamir, J. Appl. Polym. Sci. 8, 2751 (1964).

[12] R. Little and M. Wiegard, J. Appl. Polym. Sci. 14, 409 (1970).

[13] See Supplemental Material at http://link.aps.org/ supplemental/10.1103/PhysRevLett.120.124501 for a more detailed description of the experimental setup and procedures.

[14] D. Barkley, B. Song, V. Mukund, G. Lemoult, M. Avila, and B. Hof, Nature (London) 526, 550 (2015).

[15] M. Warholic, H. Massah, and T. Hanratty, Exp. Fluids 27, 461 (1999). 